\title{
SacPox from the thermoacidophilic crenarchaeon Sulfolobus acidocaldarius is a proficient lactonase
}

\author{
Janek Bzdrenga ${ }^{1 \dagger}$, Julien Hiblot ${ }^{1 \dagger}$, Guillaume Gotthard ${ }^{1}$, Charlotte Champion ${ }^{1}$, Mikael Elias ${ }^{{ }^{*}}$ and Eric Chabriere ${ }^{1 *}$
}

\begin{abstract}
Background: SacPox, an enzyme from the extremophilic crenarchaeal Sulfolobus acidocaldarius (Sac), was isolated by virtue of its phosphotriesterase (or paraoxonase; Pox) activity, i.e. its ability to hydrolyze the neurotoxic organophosphorus insecticides. Later on, SacPox was shown to belong to the Phosphotriesterase-Like Lactonase family that comprises natural lactonases, possibly involved in quorum sensing, and endowed with promiscuous, phosphotriesterase activity.

Results: Here, we present a comprehensive and broad enzymatic characterization of the natural lactonase and promiscuous organophosphorus hydrolase activities of SacPox, as well as a structural analysis using a model.

Conclusion: Kinetic experiments show that SacPox is a proficient lactonase, including at room temperature. Moreover, we discuss the observed differences in substrate specificity between SacPox and its closest homologues SsoPox and SisLac together with the possible structural causes for these observations.
\end{abstract}

Keywords: Lactonase, PLL, Quorum sensing, Phosphotriesterase, Extremophile, Thermoacidophile

\section{Background}

Phosphotriesterase-Like Lactonases (PLLs) are natural lactonases (EC 3.1.1.25) (Figure 1C, D, E) with promiscuous phosphotriesterase activity (EC 3.1.8.1) (Figure 1A) $[1,2]$. They are structurally closely related to bacterial phosphotriesterases (PTEs) [3-6], such as Brevundimonas diminuta PTE (BdPTE; 30\% sequence identity) [7]. PTEs naturally hydrolyze neurotoxic organophosphorus (OPs) compounds (Figure 1A) such as paraoxon (the active metabolite of the insecticide parathion) with catalytic constants that approach the diffusion limit (i.e. $\mathrm{k}_{\mathrm{cat}} /$ $\mathrm{K}_{\mathrm{M}} \sim 10^{8} \mathrm{M}^{-1} \mathrm{~s}^{-1}$ ) [7]. Because OPs have been massively used as pesticides since the 50's [8], PTEs are believed to have emerged in few decades from a PLL progenitor [2], providing a new source of phosphorus to bacteria, and consequently a selective advantage [8].

Both enzyme families exhibit the same $(\beta / \alpha)_{8}$-barrel topology $[9,10]$ and belong to the amidohydrolase superfamily $[11,12]$. Their structure consists of $8 \beta$-strands forming a central barrel surrounded by $8 \alpha$-helixes. The

\footnotetext{
* Correspondence: mikael.elias@gmx.fr; eric.chabriere@univ-amu.fr

${ }^{\dagger}$ Equal contributors

${ }^{2}$ Weizmann Institute of Science, Biological Chemistry, Rehovot, Israel 'URMITE UMR CNRS-IRD 6236, IFR48, Faculté de Médecine et de Pharmacie, Université de la Méditerranée, Marseille, France
}

active site is constituted by a bimetallic center (two metal cations) localized at the C-terminus of the barrel. Metal cations are coordinated by four histidines, an aspartic acid and a carboxylated lysine residue [9]. While the nature of the bimetallic center can vary depending on the enzyme nature and the purification procedure $[3,5,13,14]$, the catalytic mechanism is presumed to be identical. The bimetallic center activates a water molecule into a hydroxide ion which performs a nucleophilic attack onto the electrophilic center $[9,15]$.

The difference in substrate specificities of PLLs and PTEs seems mainly governed by variation in the connecting loops of the barrel $[2,16]$. Major differences between PTEs and PLLs reside in the active site loop size and conformation [1,2]. Indeed, loop 7 is shorter in PLLs than in PTEs whereas the loop 8 is larger, forming a hydrophobic channel that accommodates lactones aliphatic chain [9]. Loop 7/8 length and sequence also differ within the PLL family and led to the identification of two different subfamilies: PLLs-A and PLLs-B [2]. Both subfamilies exhibit different substrate specificities: PLLs-B are exclusively oxo-lactonases (Figure 1DE) whereas PLLs-A hydrolyze efficiently oxo-lactones and Acyl-Homoserine Lactones (AHLs, Figure 1C) [2]. AHLs are messenger molecules involved in a bacterial communication system 


\section{A}<smiles>[R]OP(=O)([O-])O[R]</smiles>

B<smiles>[R]OC([R])=O</smiles>

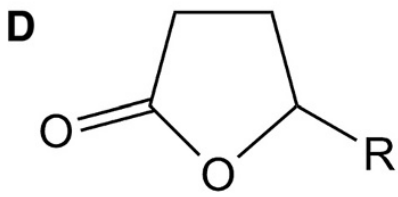

C<smiles>[R]C(=O)NC1CCOC1=O</smiles>

E<smiles>[R]C1CCCC(=O)O1</smiles>

Figure 1 Chemical structure of SacPox substrates. Chemical structures of (A) phosphotriesters, (B) esters, (C) Acyl-Homoserine Lactones, (D) $Y$-lactones and (E) $\delta$-lactones are presented. For phosphotriesters, R corresponds to different nature of substituents; LG corresponds to the leaving group. The terminal substituent could be $\mathrm{S}$ atom if the molecule is a thionophosphotriester or an $\mathrm{O}$ atom if the molecule is an oxonophosphotriester. For esters, R corresponds to different nature of substituent. For AHLs and $\gamma / \delta$-lactones, R corresponds to different size of acyl chain.

dubbed quorum sensing (QS) [17]. QS regulates the expression of numerous genes, and enables bacterial population to adopt a "group" behavior, including the expression of virulence factors of some pathogens $[18,19]$. The involvement of PLLs-A in quorum sensing has not yet been demonstrated, and these enzymes are often found with no other AHL components, including in archaeal species [20]. However, the fact that they hydrolyze specifically the natural enantiomer of AHL indicates that it may be their native substrate [16].

PLLs are promiscuous enzymes that catalyze two chemical reactions of potential biotechnological interest. Indeed, the inhibition or "quenching" of the QS is seen as a possibly promising strategy to develop innovative therapies [21-25]. Indeed, lactonases such as PLLs can inhibit QS (known as quorum quenching, i.e. QQ) [26,27] and thereby annihilate the virulence of micro-organisms possessing an AHL-based QS system [28]. Moreover, PLLs are endowed with relatively low phosphotriesterase activity, but might be optimized against OPs and subsequently used for degrading organophosphorus pesticides $[3,5,6,9,29]$ and nerve agents [30], for which no satisfactory remediation methods are currently available [31].

In addition, several PLLs members are thermostable [3,4,6,32-34]; e.g. PLLs from extremophilic crenarchaeaon sources $[3,4,16,34]$. These counterparts exhibit industrycompatible properties (e.g. thermal and detergent resistance) [35-37]; making them good starting point for in vitro improvement protocols $[37,38]$. Several studies report the engineering of thermostable PLLs and improvement of catalytic efficiency against OPs, including for SsoPox [16,39], DrOPH (Deinococcus radiodurans organophosphorus hydrolase) [6,40] and GkL (Geobacillus kaustropilus lactonase) [41] but also for the lactonase activity of SsoPox [16], MCP (Mycobacterium avium subsp. Paratuberculosis K-10 lactonase) [42] and GkL [43].

Here we focus on SacPox, the PLL from the thermoacidophilic crenarchaeon Sulfolobus acidocaldarius (living conditions: $55-85^{\circ} \mathrm{C}, \mathrm{pH} 2-3$ ) [44]. SacPox was originally isolated and studied for its ability to hydrolyze OP compounds at high temperature [4]. The enzyme shares about $30 \%$ of sequence identity with $B d \mathrm{PTE}$ and 
about 70\% with its closest homologues, i.e. SsoPox from Sulfolobus solfataricus [3] and SisLac from Sulfolobus islandicus $[33,45]$. Being an enzyme from a hyperthermophile, SacPox is however less stable than SsoPox (half-life of $5 \mathrm{~min}$ at $90^{\circ} \mathrm{C}[4]$ and of $4 \mathrm{~h}$ at $95^{\circ} \mathrm{C}[3,46]$, respectively). The kinetic characterizations performed on SacPox revealed that it hydrolyzes OP, ester and lactone molecules at high temperature $[4,13]$. However, only few substrates have been tested, and no natural lactones were assayed as substrate. In this study, we performed a broad kinetic characterization of SacPox at room temperature $\left(25^{\circ} \mathrm{C}\right)$ for several OPs, esters (Figure $1 \mathrm{~B}$ ) and lactone molecules including AHLs, $\gamma$-lactones and $\delta$-lactones in the aim to evaluate the biotechnological potentialities of this enzyme.

\section{Methods}

\section{Sequence alignment}

The sequence alignment was performed based on the previously published PLL sequence alignment [2], using the T-coffee server (expresso) [47,48] and manually improved with the seaview software [49]. It contains 29 different sequences (Additional file 1: Table S1). The sequence alignment was represented using the BioEdit 7.1.3 software [50]. Protein sequence identities were computed using ClustalW server [51]. The phylogenetic tree was performed using PhyML [49] and default parameters.

\section{Protein production and purification}

The protein production and subsequent purification steps were performed analogously to previously described [16,33,34,45,52-54]. In brief, the protein was heterologously produced in Escherichia coli strain BL21 ( $\mathrm{DE}_{3}$ )-pGro7/GroEL (TaKaRa) at $37^{\circ} \mathrm{C}$ in $\mathrm{ZYP}$ medium [55]. When $\mathrm{OD}_{600 \mathrm{~nm}}$ reaches 0.8 , protein production was induced with addition of arabinose $(0.2 \%, \mathrm{w} / \mathrm{v})$ and $\mathrm{CoCl}_{2}(2 \mathrm{mM})$ and temperature transition to $25^{\circ} \mathrm{C}$ for 20 hours. Cells were harvested by centrifugation, and pelleted cells were suspended in lysis buffer (50 mM HEPES $\mathrm{pH} 8,150 \mathrm{mM} \mathrm{NaCl}, 0.2 \mathrm{mM} \mathrm{CoCl}$, lysozyme $25 \mathrm{mg} / \mathrm{ml}$, PMSF $0.1 \mathrm{mM}$, DNase I $10 \mathrm{mg} / \mathrm{ml}$ ), stored at $-80^{\circ} \mathrm{C}$ during 2 hours; then sonicated 3 times during 30 seconds (Branson Sonifier 450, 80\% intensity and microtype limit of 8) and centrifuged. Taking advantage of the high stability of $\mathrm{SacPox}$, the supernatant was heated at $70^{\circ} \mathrm{C}$ during 30 minutes and centrifuged before proceeding a STREPTRAP affinity chromatography step (GE Healthcare, Uppsala, Sweden). The sample was then cleaved by the Tobacco Etch Virus protease (TEV, ratio 1:20, w/w [56]) during 20 hours at $30^{\circ} \mathrm{C}$ prior to be loaded a second time on STREP-TRAP affinity chromatography. The flow through containing the cleaved protein was then concentrated and loaded on a size exclusion column (S75-16-60; GE Healthcare, Uppsala, Sweden). The protein purity and identity were checked by SDS-PAGE and mass spectrometry analysis (MS platform Timone, Marseille, France). The protein concentration was determined using a nanospectrophotometer (Nanodrop, Thermofisher Scientific, France) using its molar extinction coefficient (SacPox $\varepsilon_{280 \mathrm{~nm}}=35307.7 \mathrm{M}^{-1} \mathrm{~cm}^{-1}$ ) calculated by the PROT-PARAM server [57].

\section{Kinetic characterization \\ General procedures}

Catalytic parameters were evaluated at $25^{\circ} \mathrm{C}$ and recorded with a microplate reader (Synergy HT, BioTek, USA) and the Gen5.1 software as previously explained $[16,33,52,54]$. The reaction was performed in a $200 \mu \mathrm{L}$ volume using a 96-well plate with a $6.2 \mathrm{~mm}$ path length as previously described [33]. The collected data were subsequently fitted to the Michaelis-Menten (MM) equation [58] using GraphPad Prism 5.00 (GraphPad Software, San Diego California USA, www.graphpad.com). In cases where $V_{\max }$ could not be reached, the catalytic efficiency was obtained by fitting the linear part of MM plot to a linear regression using Graph-Pad Prism 5.00 software.

\section{$O P$ hydrolase and esterase kinetics}

Standard assays for organophosphates (Figure 1A) and esters (Figure 1B) were performed in activity buffer (50 mM HEPES pH 8, $150 \mathrm{mM} \mathrm{NaCl}, 0.2 \mathrm{mM} \mathrm{CoCl}_{2}$ ) by measuring the $p$-nitrophenolate release over time at $405 \mathrm{~nm}\left(\varepsilon_{405 \mathrm{~nm}}=17000 \mathrm{M}^{-1} \mathrm{~cm}^{-1}\right)$. For ethyl-paraoxon (Additional file 1: Figure S1I), the activity buffer has also been supplemented with SDS (w/v) at $0.01 \%$ or $0.1 \%$ for detergent essays. Malathion (Additional file 1: Figure S1V) hydrolysis was followed at $412 \mathrm{~nm}$ in activity buffer added of $2 \mathrm{mM}$ DTNB to follow the release of free thiols $\left(\varepsilon_{412 \mathrm{~nm}}=13700 \mathrm{M}^{-1} \mathrm{~cm}^{-1}\right)$. The time course hydrolysis of dihydrocoumarin (Additional file 1: Figure S1X), CMPcoumarin (Additional file 1: Figure S1VI) and phenylacetate (Additional file 1: Figure S1VII) were respectively monitored at $270 \mathrm{~nm}\left(\varepsilon_{270 \mathrm{~nm}}=1400 \mathrm{M}^{-1} \mathrm{~cm}^{-1}\right), 412 \mathrm{~nm}$ $\left(\varepsilon_{412 \mathrm{~nm}}=37000 \mathrm{M}^{-1} \mathrm{~cm}^{-1}\right)$ and $270 \mathrm{~nm}\left(\varepsilon_{270 \mathrm{~nm}}=1\right.$ $\left.400 \mathrm{M}^{-1} \mathrm{~cm}^{-1}\right)$.

\section{Lactonase kinetics}

Kinetics monitoring the lactone hydrolysis were performed according to a previously described protocol [33]. The lactone hydrolysis was monitored in the lactonase buffer (2.5 mM Bicine $\mathrm{pH} 8.3,150 \mathrm{mM} \mathrm{NaCl}$, $0.2 \mathrm{mM} \mathrm{CoCl}, 0.25 \mathrm{mM}$ Cresol purple and $0.5 \%$ DMSO) with different AHLs (Figure 1C) [i.e. C4-AHL $(r)$, C6-AHL $(r)$, C8-AHL (r), 3-oxo-C8-AHL (l), 3oxo-C10-AHL $(l)]$ (Additional file 1: Figure S1XI-XVI) and oxo-lactones (Figure 1D,E) [i.e. E-caprolactone, $\gamma$-heptanolide $(r)$, Nonanoic- $\gamma$-lactone $(r)$, Nonanoic- $\delta$ lactone $(r)$, Undecanoic- $\gamma$-lactone $(r)$, Undecanoic- $\delta$ - 
A
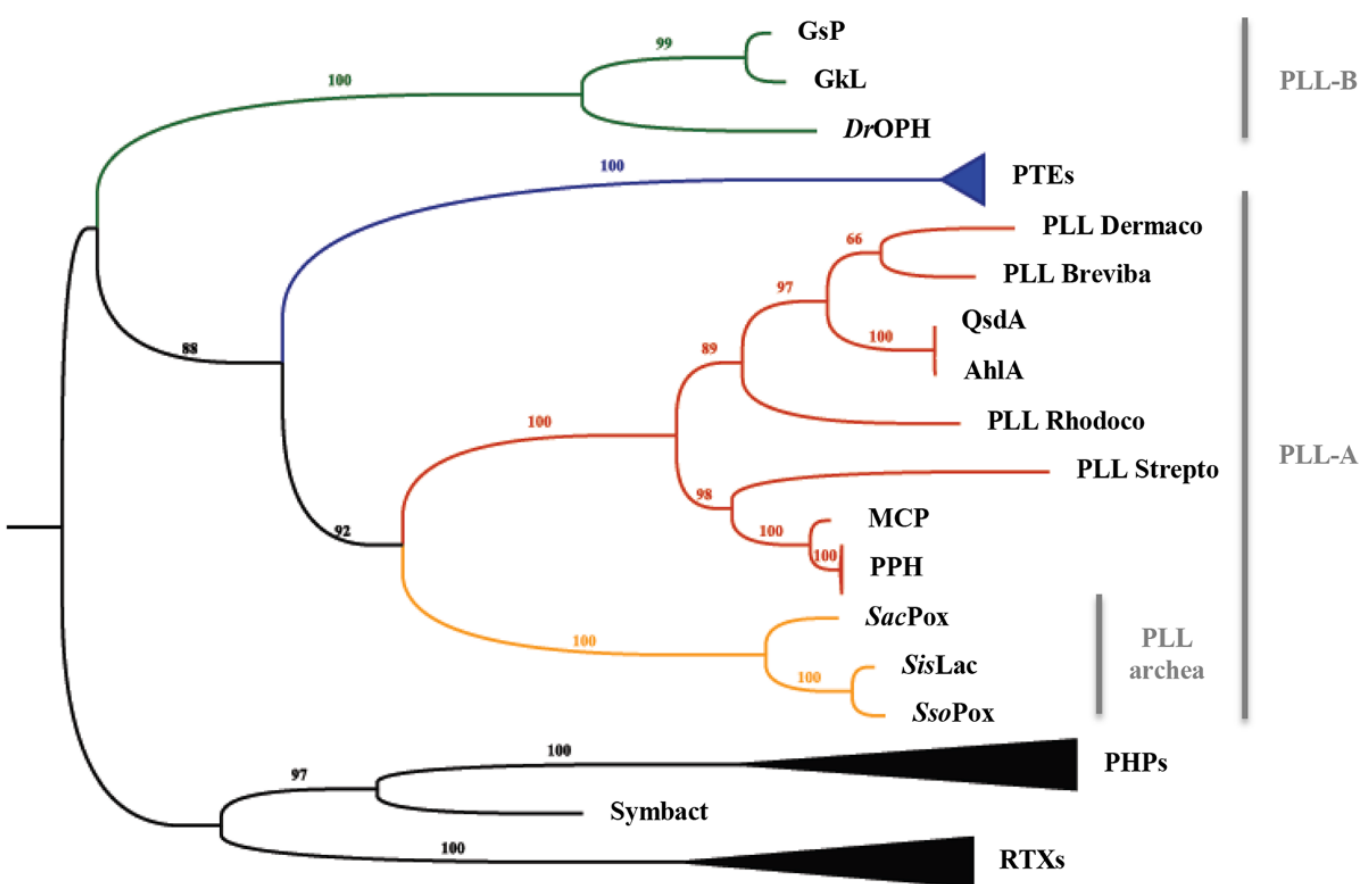

PHPs

B

0.2

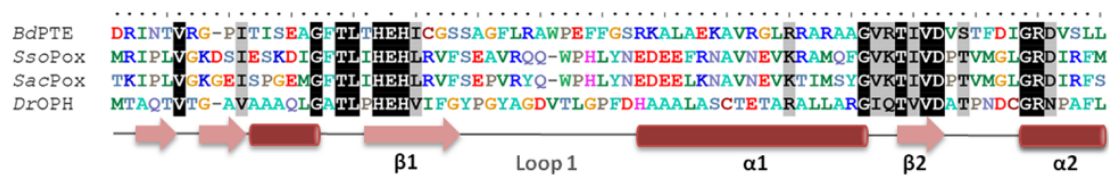

$\begin{array}{ccccccc}90 & 100 & 110 & 120 & 130 & 140 & 150\end{array}$

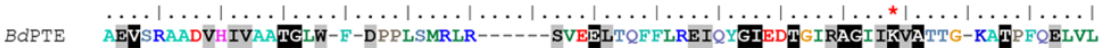

$S S O P O X$ EKVVKATG INLVAGTGIYIYIDLPFYFLNR------SIDEIADLFIHDIKEGIR GTLNAGEVKIA ADEP ITKDVEKVI

SaCPOX EKVVKETGINVIAATGLYTYTDLPFFFNGR------SLEEIAELLIHDIKKGIR GTNNRAGFIKVAADEPGITRDVERAI

DYOPH REVSEATGLOILCA TGFYYEGGATTYFKFRASL DAESEIYEMMRTEVTEGIAG IRAGVIKLASSRDAITPYEQLFF
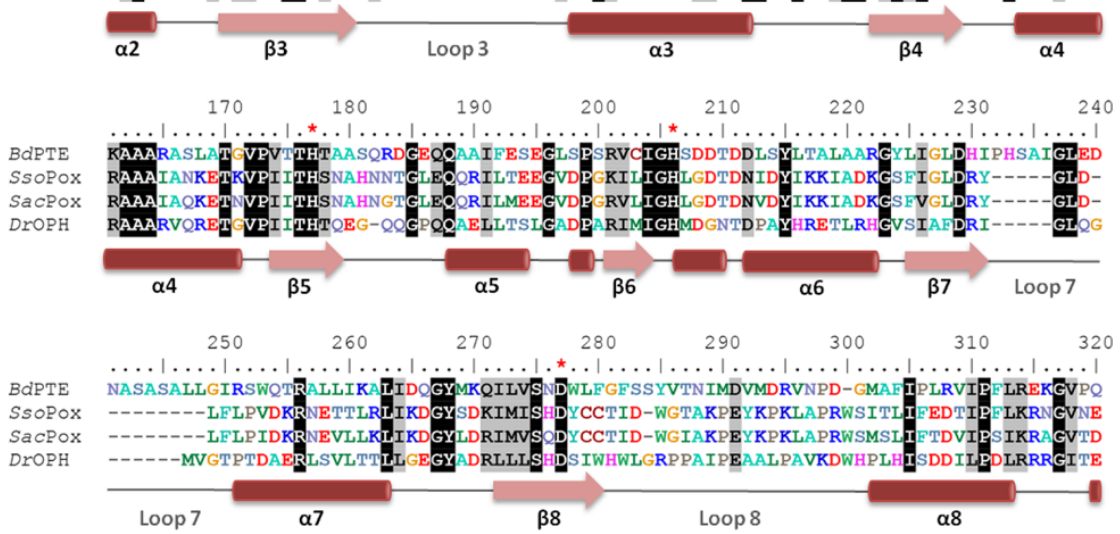

330

340

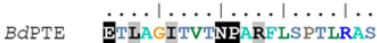

SSOPOX

SACPOX EVLHVIFVKNPARLFS-----

DrOPH BOVGOMTVGNPARLG--.-.

Figure 2 (See legend on next page.) 
(See figure on previous page.)

Figure 2 Phylogenetic analysis of the PLL family. A. Phylogenetic tree of PLLs, PTEs, and close homologues. Members of PLL-B are colored in green while within the PLL-As, mesophilic and archaeal PLLs are respectively colored in red and orange. The clades of PHPs, PTEs and RTXs were collapsed for clarity. All the sequences used for this tree are listed in Additional file 1: Table S1. B. Sequence alignment of BdPTE from B. diminuta, SsoPox from S. solfataricus, SacPox from S. acidocaldarius and DrOPH from D. radiodurans. Conserved amino acid residues are highlighted in black and similar residues in grey. Conserved active site residues involved in metals coordination are highlighted by red stars. Secondary structures are represented according to SsoPox structure (with pink arrows depicting $\beta$-sheets and red cylinders depicting a-helixes).

lactone $(r)$, Dodecanoic- $\gamma$-lactone $(r)$ and Dodecanoic$\delta$-lactone $(r)$ ] (Additional file 1: Figure S1XVII-XXIV). Cresol purple $\left(\mathrm{pK}_{\mathrm{a}} 8.3\right.$ at $\left.25^{\circ} \mathrm{C}\right)$ is a $\mathrm{pH}$ indicator $(577 \mathrm{~nm})$ used to monitor the acidification of the medium following lactone ring hydrolysis $\left(\varepsilon_{577 \mathrm{~nm}}=5\right.$ $\left.500 \mathrm{M}^{-1} \mathrm{~cm}^{-1}\right)$.

\section{Structural modeling and structural analysis}

The SacPox structure was modelled using the ESyPred3D server using SacPox protein sequence as query and SsoPox structure (2VC5) as template [59]. Structures were analyzed and figure made using PyMol [60].

\section{Results}

First classified within the bacterial PTEs, SacPox shares in fact only $33.8 \%$ sequence identity with $B d P T E$ (Additional file 1: Table S2). SacPox indeed belongs to the PLLs-A (Figure 2A) [2]: it shares $76.1 \%$ of sequence identity with its closest homologues SsoPox and SisLac, and only 30.6\% identity with the PLL-B DrOPH. Together with SisLac and SsoPox, SacPox comprises the creanarcheal clade of the PLLs-A (Figure 2A). The sequence alignment highlights the strict conservation of essential active site residues between the different clades (Figure 2B).

\section{Enzymatic characterization}

\section{Phosphotriesterase activity}

SacPox ability to hydrolyze insecticides ethyl/methyl-paraoxon, ethyl/methyl-parathion and malathion has been evaluated (Table 1). The best SacPox phosphotriester substrate, methyl-paraoxon is processed with moderate catalytic efficiency $\left(\mathrm{k}_{\mathrm{cat}} / \mathrm{K}_{\mathrm{M}}=1.10( \pm 0.17) \times 10^{3} \mathrm{M}^{-1} \cdot \mathrm{s}^{-1}\right)$, low

Table 1 Phosphotriesterase kinetic parameters

\begin{tabular}{lccc}
\hline & $\mathbf{k}_{\mathbf{c a t}}\left(\mathbf{s}^{-\mathbf{1}}\right)$ & $\mathbf{K}_{\mathbf{M}}(\boldsymbol{\mu M})$ & $\mathbf{k}_{\mathbf{c a t}} / \mathrm{K}_{\mathbf{M}}\left(\mathbf{M}^{-\mathbf{1}} \mathbf{.} \mathbf{s}^{-\mathbf{1}}\right)$ \\
\hline Paraoxon & $0.12 \pm 0.01$ & $434 \pm 54$ & $2.81( \pm 0.38) \times 10^{2}$ \\
Paraoxon 0.01\% SDS & $0.28 \pm 0.01$ & $537 \pm 48$ & $5.22( \pm 0.51) \times 10^{2}$ \\
Paraoxon 0.1\% SDS & $0.25 \pm 0.01$ & $405 \pm 21$ & $6.10( \pm 0.34) \times 10^{2}$ \\
Methyl Paraoxon & $0.31 \pm 0.02$ & $278 \pm 40$ & $1.10( \pm 0.17) \times 10^{3}$ \\
Parathion & $\mathrm{ND}$ & $\mathrm{ND}$ & $\mathrm{ND}$ \\
Methyl Parathion & $\mathrm{ND}$ & $\mathrm{ND}$ & $4.31 \pm 0.20$ \\
Malathion & $\mathrm{ND}$ & $\mathrm{ND}$ & $\mathrm{ND}$ \\
CMP-Coumarin & $0.28 \pm 0.02$ & $642 \pm 89$ & $4.38( \pm 0.68) \times 10^{2}$ \\
\hline
\end{tabular}

ND correspond to Not Detected hydrolysis. Results have been obtained with cobalt as cofactor. rate $\left(\mathrm{k}_{\mathrm{cat}}=0.307 \mathrm{~s}^{-1}\right)$ and low $\mathrm{K}_{\mathrm{M}}(278.3 \mu \mathrm{M})$. Very similar catalytic efficiencies were recorded for SsoPox and SisLac: $\mathrm{k}_{\text {cat }} / \mathrm{K}_{\mathrm{M}}$ of $1.27 \times 10^{3} \mathrm{M}^{-1} \cdot \mathrm{s}^{-1}$ and $4.26 \times 10^{3} \mathrm{M}^{-1} . \mathrm{s}^{-1}$, respectively [33,52]. Ethyl-paraoxon comprise a slower substrate, $\left(\mathrm{k}_{\text {cat }} / \mathrm{K}_{\mathrm{M}}=2.81 \times 10^{2} \mathrm{M}^{-1} \cdot \mathrm{s}^{-1}\right)$, highlighting the enzyme preference for OP substrates with small substituents. No hydrolysis could be measured for ethyl-parathion and malathion, whereas a low catalytic efficiency was recorded for methyl-parathion $\left(\mathrm{k}_{\mathrm{cat}} / \mathrm{K}_{\mathrm{M}}=4.31 \mathrm{M}^{-1} \cdot \mathrm{s}^{-1}\right)$. This specificity profile illustrates the clear preference of SacPox for oxono-phosphotriesters rather than thionophosphotriesters; as previously observed for SsoPox [52] and SisLac [33]. Moreover, whereas anionic detergents like SDS can significantly stimulate SsoPox phosphotriesterase activity [52], the same treatment on SacPox yields only a 2 -fold increase in catalytic efficiency with ethyl-paraoxon as substrate. Finally, we show that SacPox hydrolyzes CMP-coumarin $\left(\mathrm{k}_{\mathrm{cat}} / \mathrm{K}_{\mathrm{M}}=4.38 \times 10^{2} \mathrm{M}^{-1} \cdot \mathrm{s}^{-1}\right)$, albeit with 20-fold lower catalytic efficiency than SsoPox [52].

\section{Esterase activity}

The ability of SacPox to hydrolyze phenyl-acetate, $p$ NPacetate and $p \mathrm{NP}$-decanoate (Additional file 1: Figure S1VII-IX) has been evaluated (Table 2). While no activity could be detected against $p$ NP-decanoate, SacPox exhibits low catalytic efficiencies against both phenyl-acetate and $p$ NP-acetate $\left(\mathrm{k}_{\text {cat }} / \mathrm{K}_{\mathrm{M}} \approx 50 \mathrm{M}^{-1} \cdot \mathrm{s}^{-1}\right)$. This weak activity against classical esters differs from previous studies on the close homologues SsoPox and SisLac, for which activity has only been recorded on $p \mathrm{NP}$-acetate [33].

\section{Lactonase activity}

The catalytic parameters of SacPox for various lactone substrates have been measured, including against oxolactones (lipophilic aroma), AHLs and dihydrocoumarin (Table 3). Our results indicate a preference of SacPox for oxo-lactone substrates; i.e. $\gamma$-heptanolide and nonanoic- $\gamma$ lactone $\left(\mathrm{k}_{\mathrm{cat}} / \mathrm{K}_{\mathrm{M}} \approx 2.5 \times 10^{4} \mathrm{M}^{-1} \cdot \mathrm{s}^{-1}\right)$, while AHLs are about 10 times worse substrates (i.e.; C8 AHLs, $\mathrm{k}_{\text {cat }} / \mathrm{K}_{\mathrm{M}} \approx 5 \times 10^{3}$ $\left.\mathrm{M}^{-1} \cdot \mathrm{s}^{-1}\right)$. Furthermore, it seems that SacPox prefers AHLs vs 3-oxo-AHLs since the $\mathrm{K}_{\mathrm{M}}$ for $\mathrm{C} 8$ aliphatic chains is 5fold lower than that for 3-oxo-C8 AHLs. Overall, long aliphatic chain substrates AHLs are better substrates for the enzyme. Indeed, short aliphatic chain AHLs are not hydrolyzed by SacPox. Interestingly, this preference is not retained for oxo-lactones, for which molecules with short 
Table 2 Esterase kinetic parameters

\begin{tabular}{lccc}
\hline & $\mathbf{k}_{\mathrm{cat}}\left(\mathbf{s}^{-\mathbf{1}}\right)$ & $\mathbf{K}_{\mathbf{M}}(\boldsymbol{\mu M})$ & $\mathbf{k}_{\mathrm{cat}} / \mathbf{K}_{\mathbf{M}}\left(\mathbf{M}^{-\mathbf{1}} \cdot \mathbf{s}^{-\mathbf{1}}\right)$ \\
\hline Phenyl-acetate & $0.35 \pm 0.05$ & $8181 \pm 1750$ & $42.3 \pm 11.1$ \\
$\boldsymbol{p}$ NP-acetate & $0.13 \pm 0.01$ & $2107 \pm 313$ & $60.1 \pm 9.9$ \\
$\boldsymbol{p N P}$-decanoate & $\mathrm{ND}$ & $\mathrm{ND}$ & $\mathrm{ND}$
\end{tabular}

ND correspond to Not Detected hydrolysis. Results have been obtained with cobalt as cofactor.

or without aliphatic chain are efficiently hydrolyzed $\left(\mathrm{k}_{\text {cat }}\right)$ $\left.\mathrm{K}_{\mathrm{M}} \approx 10^{4} \mathrm{M}^{-1} \cdot \mathrm{s}^{-1}\right)$. As previously observed for SsoPox and SisLac $[16,33]$, this feature may reveal a potential alternative binding mode of these compounds in SacPox active site. Finally, contrary to SsoPox and SisLac [16,33], SacPox does not hydrolyze dihydrocoumarin.

\section{Structural analysis}

Numerous attempts to crystallize SacPox were made, with no success (Elias, Hiblot, Gotthard \& Chabriere, unpublished). A previous structural model was generated by homology modeling based on BdPTE structure [4] ( 33.8\% sequence identity with $\mathrm{SacPox}$ ), but yielded little insights given the moderate sequence identity with the template and the very significant differences in the active site loops between these two representatives of distinct enzyme families $[1,9,16]$. Here we generated a homology-based model using the structure of SsoPox as template $(76.1 \%$ of sequence identity; Additional file 1: Table S2).

As expected, the SacPox model structure almost perfectly superimposes to the SsoPox crystal structure (Figure 3A). Residues forming the active site are all conserved and residues involved in loops 7 and 8 occupy nearly identical conformation in SacPox and SsoPox but

Table 3 Lactonase kinetic parameters

\begin{tabular}{|c|c|c|c|}
\hline & $\mathrm{k}_{\text {cat }}\left(\mathrm{s}^{-1}\right)$ & $\mathrm{K}_{\mathrm{M}}(\mu \mathrm{M})$ & $\mathrm{k}_{\text {cat }} / \mathrm{K}_{\mathrm{M}}\left(\mathrm{M}^{-1} \cdot \mathrm{s}^{-1}\right)$ \\
\hline C4 AHL & ND & ND & ND \\
\hline $\mathrm{C} 6 \mathrm{AHL}$ & ND & ND & ND \\
\hline C8 AHL & $0.94 \pm 0.02$ & $178 \pm 26$ & $5.28( \pm 0.77) \times 10^{3}$ \\
\hline 3-0xo C6 AHL & ND & ND & ND \\
\hline 3-oxo C8 AHL & $0.89 \pm 0.07$ & $836 \pm 178$ & $1.07( \pm 0.25) \times 10^{3}$ \\
\hline 3-oxo C10 AHL & $1.03 \pm 0.04$ & $213 \pm 33$ & $4.88( \pm 0.77) \times 10^{3}$ \\
\hline Y heptanolide & $10.25 \pm 0.50$ & $388 \pm 62$ & $2.64( \pm 0.44) \times 10^{4}$ \\
\hline Nonanoic- $\gamma$-lactone & $2.64 \pm 0.07$ & $109 \pm 19$ & $2.44( \pm 0.44) \times 10^{4}$ \\
\hline Undecanoic- $\gamma$-lactone & $0.34 \pm 0.01$ & $578 \pm 78$ & $5.89( \pm 0.84) \times 10^{2}$ \\
\hline dodecanoic- $\gamma$-lactone & $0.53 \pm 0.03$ & $242 \pm 60$ & $2.21( \pm 0.57) \times 10^{3}$ \\
\hline Nonanoic- $\delta$-lactone & $4.55 \pm 0.21$ & $348 \pm 53$ & $1.31( \pm 0.21) \times 10^{4}$ \\
\hline Undecanoic- $\delta$-lactone & $1.05 \pm 0.05$ & $168 \pm 37$ & $6.22( \pm 1.40) \times 10^{3}$ \\
\hline Dodecanoic- $\delta$-lactone & $3.34 \pm 0.07$ & $185 \pm 27$ & $1.81( \pm 0.27) \times 10^{4}$ \\
\hline$\varepsilon$ caprolactone & $15.04 \pm 0.47$ & $1031 \pm 83$ & $1.46( \pm 0.13) \times 10^{4}$ \\
\hline Dihydrocoumarine & ND & ND & ND \\
\hline
\end{tabular}

ND correspond to Not Detected hydrolysis. Results have been obtained with cobalt as cofactor. also in SisLac structures (Figure 3B). Noteworthy, loop 8 is partially structured into an $\alpha$-helix, as seen in X-ray structures of SsoPox and SisLac. A substitution (I266 in SacPox; T265 in SsoPox and SisLac) in loop 8 may slightly alter the shape of the aliphatic channel. But overall, the active site of SacPox and SsoPox are nearly identical (Figure 2B). Furthermore, four other substitutions between SacPox and its close homologues can be seen in loop 8: SacPox exhibits a K at position 268, instead of an R residue (R267 in SisLac), Y271 instead of L (L270 in SisLac), K278 instead of R (R277 in both SisLac and SsoPox), and M281 instead of I (I280 in SsoPox) (Additional file 1: Figure S2). While the structural model suggests that these substitutions are not affecting directly the binding cleft of SacPox, they might modulate loop 8 conformation and its dynamics. Indeed, it was shown in the close homologue SsoPox that a single substitution in loop 8 (W263 in SsoPox, equivalent to W264 in SacPox) increases the conformational flexibility of loop 8 , thereby conferring higher promiscuity to the enzyme [16]. The effect is in fact so dramatic that the substitution in SsoPox of W263 by any of the 19 other natural amino acids yields a variant with improved phosphotriesterase activity [16]. Additionally, loop 8 being involved in the accommodation of the aliphatic substituent of lactones substrates [9], mutations in this loop can also affect the lactonase activity [16].

\section{Discussion}

Here we show that SacPox is a proficient lactonase $\left(\sim 10^{4} \mathrm{M}^{-1} \cdot \mathrm{s}^{-1}\right)$ and can hydrolyze both oxo-lactones and AHLs. Nevertheless, SacPox have a slightly different substrate specificity than its close homologues [16,33]. Indeed, SacPox exhibits slightly lower catalytic efficiencies, prefers AHLs over 3-oxo-AHLs and does not show any activity against dihydrocoumarin. Interestingly, as noted for SisLac and SsoPox [16,33], SacPox clearly prefers long chain AHLs, but can efficiently hydrolyze short chain or oxo-lactones without aliphatic substituents. This feature could reflect a putatively different binding mode of AHLs and oxo-lactones into PLLs active sites. We note that the biological role of lactonases such as PLLs is yet unclear, especially in extremophilic archaea where no AHL-based quorum sensing systems have been identified so far.

SacPox also exhibits promiscuous esterase and phosphotriesterase activities, a common feature of PLLs. Similarly to SsoPox and SisLac [33,52], SacPox prefers OPs with small substituents. Moreover, SacPox also shows a clear preference for oxono-phosphotriesters, rather than thionophosphotriesters, a feature previously dubbed thiono-effect [52]. Interestingly, SsoPox, SisLac and SacPox exhibit similar catalytic efficiencies against OPs $\left(10^{2-3} \mathrm{M}^{-1} \cdot \mathrm{s}^{-1}\right)$ at $25^{\circ} \mathrm{C}$, efficiencies that are close to those measured at much higher temperatures [4]. 

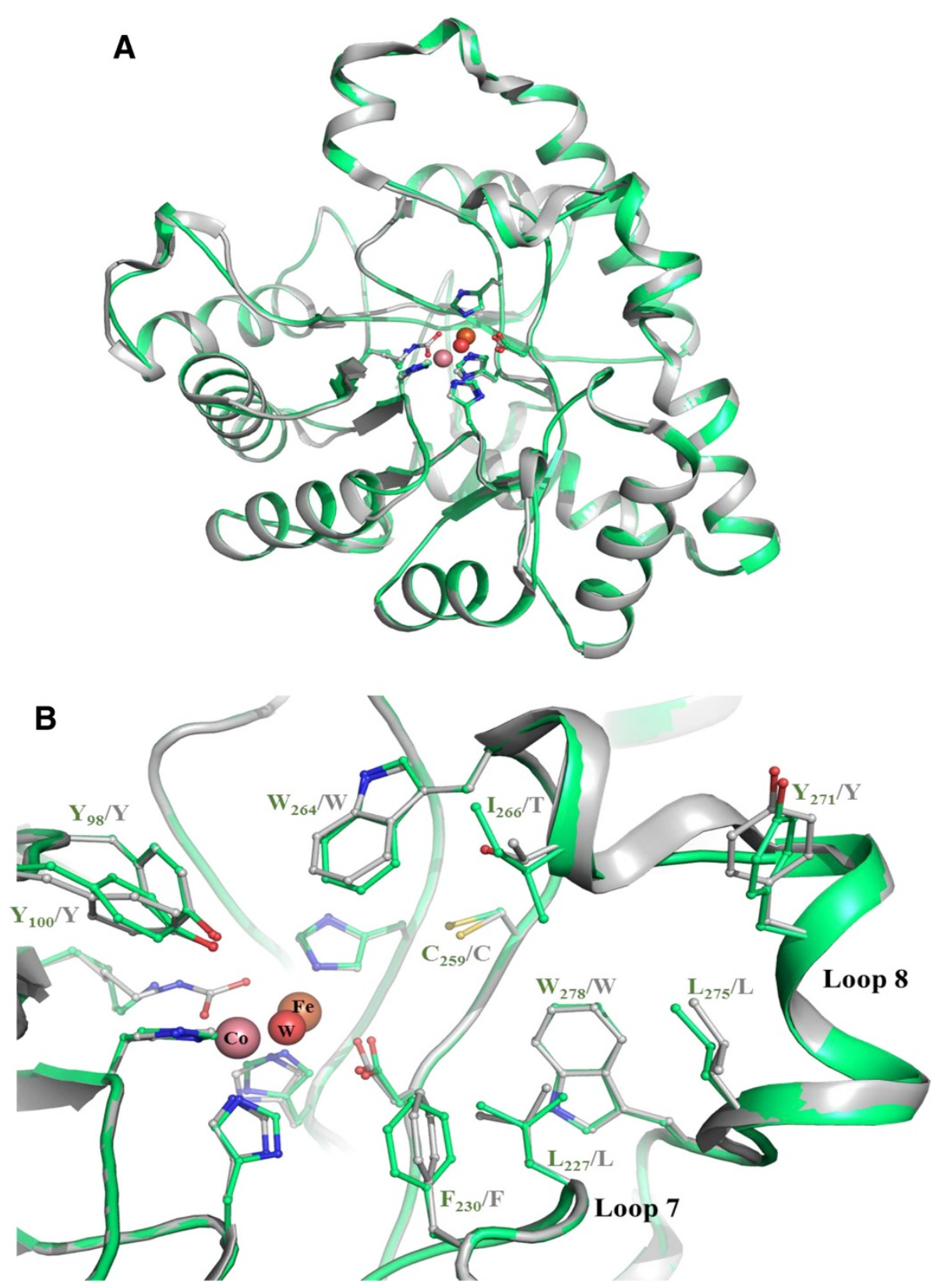

Figure 3 Structural model of SacPox. A. Structural superposition of SsoPox structure (2VC5; grey) and the SacPox model (green). Cobalt, iron and the catalytic water molecule are respectively represented by pink, orange and red spheres. Bimetallic center coordinating residues are represented as sticks. B. Active site view of superimposed SsoPox structure (grey) and the SacPox model (green). Several active site residues are represented as sticks. Numbering is made according to SacPox sequence.

The structural model shows that SacPox structure is very close to that of SsoPox (Figure 2A). Most critically, the active sites of both enzymes are essentially identical (Figure 2B), with the exception of position 266 (I in SacPox, T in SsoPox and SisLac). This substitution might partly account for the observed differences in substrates specificity between these enzymes, and would thereby represent an interesting target for future mutagenesis studies. But four other substitutions in loop 8 between these close homologues might be involved as well, and comprise also interesting options for mutagenesis studies (K268R, Y27IL, K278R and M281I). A recent study on SsoPox highlighted how profound the effect on catalysis of a single substitution on loop 8 (W263) can be [16]. Therefore, substitution T266I, and/or the four others on loop 8, might contribute to the observed differences between SacPox and SsoPox in substrate specificity, in combination with other factors that cannot be assessed 
by a structural model such as subtle changes in active site loops conformation and dynamics $[16,33]$. Indeed, the observed differences in the detergent stimulation between both enzymes (SacPox is only weakly stimulated by SDS, as compared to SsoPox) could well be a manifestation of different dynamics of their respective active site loops.

\section{Conclusions}

To conclude, we here demonstrate that albeit being initially isolated, characterized, and named after its ability to degrade the insecticide paraoxon (pox; [4]), SacPox is putatively a native lactonase, capable of hydrolyzing these compounds with significant catalytic efficiencies at $25^{\circ} \mathrm{C}$ (up to $10^{4} \mathrm{M}^{-1} \cdot \mathrm{s}^{-1}$ ). The extensive kinetic characterization reveals some substrate specificity differences between $\mathrm{Sac}$ Pox and its close homologues SisLac and SsoPox, and the proposed structural model of SacPox suggests putative candidates (e.g. I266) that could account for these observations. Such positions might constitute interesting targets for future engineering studies, with the aim of improving or altering the catalytic properties of SacPox.

\section{Additional file}

Additional file 1: Figure S1. Chemical structure of phosphoesters (I-VI), esters (VII-IX) and lactones (X-XXIV). Figure S2. Superposition of SsoPox, SisLac and SacPox structural models. Table S1. Accession numbers of the sequences used in the phylogeny study. Table S2. Sequence identity matrix.

\section{Competing interests}

The authors declare that they have no competing interests.

\section{Authors' contributions}

$J H, G G$ and ME planed the experiments. JB, CC performed the experiments. $J H, J B, M E$ and $E C$ analysed the results. $J B, J H$ and ME wrote the paper. All the authors offered a critical review of the paper.

\section{Acknowledgements}

We are grateful to Dr. Moshe Goldsmith for the kind gift of CMP-coumarin. This work was granted by DGA, France (REl. 200934 0045). J.B. is a PhD student granted by DGA. J.H. and C.C. are founded by DGA, France. G.G. is founded by APHM, France.

Received: 4 February 2014 Accepted: 27 May 2014

Published: 3 June 2014

\section{References}

1. Afriat L, Roodveldt C, Manco G, Tawfik DS: The latent promiscuity of newly identified microbial lactonases is linked to a recently diverged phosphotriesterase. Biochemistry 2006, 45:13677-13686.

2. Afriat-Jurnou L, Jackson CJ, Tawfik DS: Reconstructing a missing link in the evolution of a recently diverged phosphotriesterase by active-site loop remodeling. Biochemistry 2012, 51:6047-6055.

3. Merone L, Mandrich L, Rossi M, Manco G: A thermostable phosphotriesterase from the archaeon Sulfolobus solfataricus: cloning, overexpression and properties. Extremophiles 2005, 9:297-305.

4. Porzio E, Merone L, Mandrich L, Rossi M, Manco G: A new phosphotriesterase from Sulfolobus acidocaldarius and its comparison with the homologue from Sulfolobus solfataricus. Biochimie 2007, 89:625-636.

5. Xiang DF, Kolb P, Fedorov AA, Meier MM, Fedorov LV, Nguyen TT, Sterner R, Almo SC, Shoichet BK, Raushel FM: Functional annotation and three-dimensional structure of Dr0930 from Deinococcus radiodurans, a close relative of phosphotriesterase in the amidohydrolase superfamily. Biochemistry 2009, 48:2237-2247.

6. Hawwa R, Larsen SD, Ratia K, Mesecar AD: Structure-based and random mutagenesis approaches increase the organophosphate-degrading activity of a phosphotriesterase homologue from Deinococcus radiodurans. J Mol Biol 2009, 393:36-57.

7. Dumas DP, Caldwell SR, Wild JR, Raushel FM: Purification and properties of the phosphotriesterase from Pseudomonas diminuta. J Biol Chem 1989, 264:19659-19665.

8. Singh BK: Organophosphorus-degrading bacteria: ecology and industrial applications. Nat Rev Microbiol 2009, 7:156-164.

9. Elias M, Dupuy J, Merone L, Mandrich L, Porzio E, Moniot S, Rochu D, Lecomte C, Rossi M, Masson P, Manco G, Chabriere E: Structural basis for natural lactonase and promiscuous phosphotriesterase activities. J Mol Biol 2008, 379:1017-1028.

10. Benning MM, Kuo JM, Raushel FM, Holden HM: Three-dimensional structure of phosphotriesterase: an enzyme capable of detoxifying organophosphate nerve agents. Biochemistry 1994, 33:15001-15007.

11. Seibert CM, Raushel FM: Structural and catalytic diversity within the amidohydrolase superfamily. Biochemistry 2005, 44:6383-6391.

12. Roodveldt C, Tawfik DS: Shared promiscuous activities and evolutionary features in various members of the amidohydrolase superfamily. Biochemistry 2005, 44:12728-12736.

13. Porzio E, Di Gennaro S, Palma A, Manco G: Mn(2+) modulates the kinetic properties of an archaeal member of the PLL family. Chem Biol Interact 2013, 203:251-256.

14. Xue B, Chow JY, Baldansuren A, Yap LL, Gan YH, Dikanov SA, Robinson RC, Yew WS: Correction to structural evidence of a productive active site architecture for an evolved quorum-quenching GKL lactonase. Biochemistry 2012, 51:10120.

15. Bigley AN, Raushel FM: Catalytic mechanisms for phosphotriesterases. Biochim Biophys Acta 2013, 1834:443-453.

16. Hiblot J, Gotthard G, Elias M, Chabriere E: Differential active site loop conformations mediate promiscuous activities in the lactonase pox. PLoS One 2013, 8:e75272.

17. Waters CM, Bassler BL: Quorum sensing: cell-to-cell communication in bacteria. Annu Rev Cell Dev Biol 2005, 21:319-346.

18. Popat R, Crusz SA, Diggle SP: The social behaviours of bacterial pathogens. Br Med Bull 2008, 87:63-75.

19. Boyen F, Eeckhaut V, Van Immerseel F, Pasmans F, Ducatelle R, Haesebrouck F: Quorum sensing in veterinary pathogens: mechanisms, clinical importance and future perspectives. Vet Microbiol 2009, 135:187-195.

20. Elias M, Tawfik DS: Divergence and convergence in enzyme evolution: parallel evolution of paraoxonases from quorum-quenching lactonases. J Biol Chem 2012, 287:11-20.

21. Amara N, Krom BP, Kaufmann GF, Meijler MM: Macromolecular inhibition of quorum sensing: enzymes, antibodies, and beyond. Chem Rev 2011, 111:195-208.

22. Hentzer M, Wu H, Andersen JB, Riedel K, Rasmussen TB, Bagge N, Kumar N, Schembri MA, Song Z, Kristoffersen P, Manefield M, Costerton JW, Molin S, Eberl L, Steinberg P, Kjelleberg S, Høiby N, Givskov M: Attenuation of Pseudomonas aeruginosa virulence by quorum sensing inhibitors. EMBO J 2003, 22:3803-3815.

23. O'Loughlin CT, Miller LC, Siryaporn A, Drescher K, Semmelhack MF, Bassler BL: A quorum-sensing inhibitor blocks Pseudomonas aeruginosa virulence and biofilm formation. Proc Natl Acad Sci U S A 2013, 110:17981-17986.

24. Wu H, Song Z, Hentzer M, Andersen JB, Molin S, Givskov M, Hoiby N Synthetic furanones inhibit quorum-sensing and enhance bacterial clearance in Pseudomonas aeruginosa lung infection in mice. J Antimicrob Chemother 2004, 53:1054-1061.

25. Christensen LD, van Gennip M, Jakobsen TH, Alhede M, Hougen HP, Hoiby N, Bjarnsholt T, Givskov M: Synergistic antibacterial efficacy of early combination treatment with tobramycin and quorum-sensing inhibitors against Pseudomonas aeruginosa in an intraperitoneal foreign-body infection mouse model. J Antimicrob Chemother 2012, 67:1198-1206.

26. Dong $Y H$, Wang $L H, X u J L$, Zhang $H B$, Zhang XF, Zhang LH: Quenching quorum-sensing-dependent bacterial infection by an $\mathrm{N}$-acyl homoserine lactonase. Nature 2001, 411:813-817.

27. Dong $Y H$, Wang $L Y$, Zhang $L H:$ Quorum-quenching microbial infections: mechanisms and implications. Philos Trans R Soc Lond B Biol Sci 2007, 362:1201-1211. 
28. Ng FS, Wright DM, Seah SY: Characterization of a phosphotriesterase-like lactonase from Sulfolobus solfataricus and its immobilization for quorum quenching. App/ Environ Microbiol 2011, 77:1181-1186.

29. Krieger Rl: Handbook of Pesticide Toxicology. 2nd edition. San Diego: Academic Press; 2001.

30. Gupta RC: Handbook of Toxicology of Chemical Warfare Agents. San Diego: Elsevier Inc; 2009.

31. LeJeune KE, Wild JR, Russell AJ: Nerve agents degraded by enzymatic foams. Nature 1998, 395:27-28.

32. Hawwa R, Aikens J, Turner RJ, Santarsiero BD, Mesecar AD: Structural basis for thermostability revealed through the identification and characterization of a highly thermostable phosphotriesterase-like lactonase from Geobacillus stearothermophilus. Arch Biochem Biophys 2009, 488:109-120.

33. Hiblot J, Gotthard G, Chabriere E, Elias M: Structural and enzymatic characterization of the lactonase SisLac from Sulfolobus islandicus. PLoS One 2012, 7:e47028.

34. Hiblot J, Gotthard G, Champion C, Chabriere E, Elias M: Crystallization and preliminary X-ray diffraction analysis of the lactonase VmoLac from Vulcanisaeta moutnovskia. Acta Crystallogr Sect F Struct Biol Cryst Commun 2013, 69:1235-1238.

35. Vieille C, Zeikus GJ: Hyperthermophilic enzymes: sources, uses, and molecular mechanisms for thermostability. Microbiol Mol Biol Rev 2001, 65:1-43.

36. Demirjian DC, Moris-Varas F, Cassidy CS: Enzymes from extremophiles. Curr Opin Chem Biol 2001, 5:144-151.

37. Burton SG, Cowan DA, Woodley JM: The search for the ideal biocatalyst. Nat Biotechnol 2002, 20:37-45.

38. Singh RK, Tiwari MK, Singh R, Lee JK: From protein engineering to immobilization: promising strategies for the upgrade of industria enzymes. Int J Mol Sci 2013, 14:1232-1277.

39. Merone L, Mandrich L, Porzio E, Rossi M, Muller S, Reiter G, Worek F, Manco G: Improving the promiscuous nerve agent hydrolase activity of a thermostable archaeal lactonase. Bioresour Technol 2010, 101:9204-9212.

40. Meier MM, Rajendran C, Malisi C, Fox NG, Xu C, Schlee S, Barondeau DP, Hocker B, Sterner R, Raushel FM: Molecular engineering of organophosphate hydrolysis activity from a weak promiscuous lactonase template. J Am Chem Soc 2013, 135:11670-11677.

41. Zhang Y, An J, Ye W, Yang G, Qian ZG, Chen HF, Cui L, Feng Y: Enhancing the promiscuous phosphotriesterase activity of a thermostable lactonase (GkaP) for the efficient degradation of organophosphate pesticides. Appl Environ Microbiol 2012, 78:6647-6655.

42. Chow JY, Wu L, Yew WS: Directed evolution of a quorum-quenching lactonase from Mycobacterium avium subsp. paratuberculosis K-10 in the amidohydrolase superfamily. Biochemistry 2009, 48:4344-4353.

43. Chow JY, Xue B, Lee KH, Tung A, Wu L, Robinson RC, Yew WS: Directed evolution of a thermostable quorum-quenching lactonase from the amidohydrolase superfamily. J Biol Chem 2010, 285:40911-40920.

44. Auernik KS, Cooper CR, Kelly RM: Life in hot acid: pathway analyses in extremely thermoacidophilic archaea. Curr Opin Biotechnol 2008, 19:445-453.

45. Gotthard G, Hiblot J, Elias M, Chabriere E: Crystallization and preliminary X-ray diffraction analysis of the hyperthermophilic Sulfolobus islandicus lactonase. Acta Crystallogr Sect F Struct Biol Cryst Commun 2011, 67:354-357.

46. Del Vecchio P, Elias M, Merone L, Graziano G, Dupuy J, Mandrich L, Carullo P, Fournier B, Rochu D, Rossi M, Masson P, Chabriere E, Manco G: Structural determinants of the high thermal stability of SsoPox from the hyperthermophilic archaeon Sulfolobus solfataricus. Extremophiles 2009, 13:461-470.

47. Notredame C, Higgins DG, Heringa J: T-Coffee: a novel method for fast and accurate multiple sequence alignment. J Mol Biol 2000, 302:205-217.

48. Poirot O, OToole E, Notredame C: Tcoffee@igs: a web server for computing, evaluating and combining multiple sequence alignments. Nucleic Acids Res 2003, 31:3503-3506.

49. Gouy M, Guindon S, Gascuel O: SeaView version 4: a multiplatform graphical user interface for sequence alignment and phylogenetic tree building. Mol Biol Evol 2010, 27:221-224.

50. Hall TA: BioEdit: a user-friendly biological sequence alignment editor and analysis program for Windows 95/98/NT. Nucleic Acids Symposium Series 1999, 41:95-98.
51. Larkin MA, Blackshields G, Brown NP, Chenna R, McGettigan PA, McWilliam H, Valentin F, Wallace IM, Wilm A, Lopez R, Thompson JD, Gibson TJ, Higgins DG: Clustal W and Clustal X version 2.0. Bioinformatics 2007, 23:2947-2948.

52. Hiblot J, Gotthard G, Chabriere E, Elias M: Characterisation of the organophosphate hydrolase catalytic activity of SsoPox. Sci Rep 2012, 2:779.

53. Gotthard G, Hiblot J, Gonzalez D, Chabriere E, Elias M: Crystallization and preliminary X-ray diffraction analysis of the organophosphorus hydrolase OPHC2 from Pseudomonas pseudoalcaligenes. Acta Crystallogr Sect F Struct Biol Cryst Commun 2013, 69:73-76.

54. Gotthard G, Hiblot J, Gonzalez D, Elias M, Chabriere E: Structural and enzymatic characterization of the phosphotriesterase OPHC2 from Pseudomonas pseudoalcaligenes. PLoS One 2013, 8:e77995.

55. Studier FW: Protein production by auto-induction in high density shaking cultures. Protein Expr Purif 2005, 41:207-234

56. van den Berg S, Lofdahl PA, Hard T, Berglund H: Improved solubility of TEV protease by directed evolution. J Biotechnol 2006, 121:291-298.

57. Wilkins MR, Gasteiger E, Bairoch A, Sanchez JC, Williams KL, Appel RD, Hochstrasser DF: Protein identification and analysis tools in the ExPASy server. Methods Mol Biol 1999, 112:531-552.

58. Copeland RA: Enzymes, A Practical Introduction to Structure, Mechanism, and Data Analysis. 2nd edition. New York, Chichester, Weiheim, Brisbane, Singapore, Toronto: WILEY-VCH; 2000.

59. Lambert C, Leonard N, De Bolle X, Depiereux E: ESyPred3D: prediction of proteins 3D structures. Bioinformatics 2002, 18:1250-1256.

60. DeLano W: The PyMOL Molecular Graphics System. San Carlos, CA, USA: DeLano Scientific; 2002

doi:10.1186/1756-0500-7-333

Cite this article as: Bzdrenga et al: SacPox from the thermoacidophilic crenarchaeon Sulfolobus acidocaldarius is a proficient lactonase. BMC Research Notes 2014 7:333.

\section{Submit your next manuscript to BioMed Central and take full advantage of:}

- Convenient online submission

- Thorough peer review

- No space constraints or color figure charges

- Immediate publication on acceptance

- Inclusion in PubMed, CAS, Scopus and Google Scholar

- Research which is freely available for redistribution 